\title{
Leveraging Disturbance Observer Based Torque Control for Improved Impedance Rendering with Series Elastic Actuators
}

\author{
Joshua S. Mehling, James Holley, and Marcia K. O’Malley
}

\begin{abstract}
The fidelity with which series elastic actuators (SEAs) render desired impedances is important. Numerous approaches to SEA impedance control have been developed under the premise that high-precision actuator torque control is a prerequisite. Indeed, the design of an inner torque compensator has a significant impact on actuator impedance rendering. The disturbance observer (DOB) based torque control implemented in NASA's Valkyrie robot is considered here and a mathematical model of this torque control, cascaded with an outer impedance compensator, is constructed. While previous work has examined the impact a disturbance observer has on torque control performance, little has been done regarding DOBs and impedance rendering accuracy. Both simulation and a series of experiments are used to demonstrate the significant improvements possible in an SEA's ability to render desired dynamic behaviors when utilizing a DOB. Actuator transparency at low impedances is improved, closed loop hysteresis is reduced, and the actuator's dynamic response to both commands and interaction torques more faithfully matches that of the desired model. All of this is achieved by leveraging DOB based control rather than increasing compensator gains, thus making improved SEA impedance control easier to achieve in practice.
\end{abstract}

\section{INTRODUCTION}

Impedance control has seen widespread adoption in robotic systems as a means to manage contact and perform interaction tasks. As first described in [1], rather than modulating either a robot's position or force, a desired dynamic relationship between these two quantities can be defined and actively controlled. Research suggests that the human central nervous system relies on a similar control paradigm to manage interaction [2], [3]. Thus, effective impedance control can be of particular value in robots intended to perform human-like tasks or interact directly with people.

Whereas impedance control addresses the difficulty of performing interaction tasks through appropriate controller design, series elastic actuation provides a distinctly hardwarebased solution to many of the same problems. A series elastic actuator (SEA) incorporates an intentionally compliant element in series with its drivetrain and in so doing decreases the actuator's passive output impedance, improving contact stability. Extensively discussed in [4], [5], and [6], series elastic actuation can benefit shock tolerance, energy storage, and power output, while also providing an easier method to

J. S. Mehling is with the Robotic Systems Technology Branch, NASA/Johnson Space Center, Houston, TX 77058 USA and the Department of Mechanical Engineering, Rice University, Houston, TX 77005 USA joshua.s.mehling@nasa.gov

J. Holley is with the Robotic Systems Technology Branch, NASA/Johnson Space Center, Houston, TX 77058 USA james.j.holley@nasa.gov

M. K. O'Malley is with the Department of Mechanical Engineering, Rice University, Houston, TX 77005 USA omalleymerice.edu sense actuator torque (via a spring deflection measurement). The ease with which impedance controlled SEAs can actively render low output impedances is also particularly valuable in haptics, rehabilitation robotics, and human augmentation systems (e.g. [7], [8], and [9]) where importance is placed on achieving both safety and device transparency for the user.

A variety of impedance control architectures have been proposed for series elastic actuators. Most of these accept the premise that to achieve effective impedance control, highprecision actuator torque (or force) control is a prerequisite. Variation then lies in how this high-precision torque control is achieved. PID architectures (or subsets P, PD, etc.) that rely on measured spring force have been used, and are at times coupled with model based feedforward terms [4], [10]. Cascaded control structures wrapping actuator torque control around an inner position or velocity loop have also been employed [11], [12], [13], [14]; while effective SEA torque tracking has been demonstrated with disturbance observer based torque control [15], [16].

Building on previous research at the Johnson Space Center with Robonaut 2 [17], NASA's Valkyrie robot (Fig. 1) incorporates 25 series elastic actuators throughout its arms, legs, and torso to further explore the application of series elastic actuation in robots designed to do useful work in human environments. As outlined in [18], a disturbance observer (DOB) based torque control architecture has been adopted for each of these actuators. An outer impedance loop can be cascaded with Valkyrie's DOB based torque

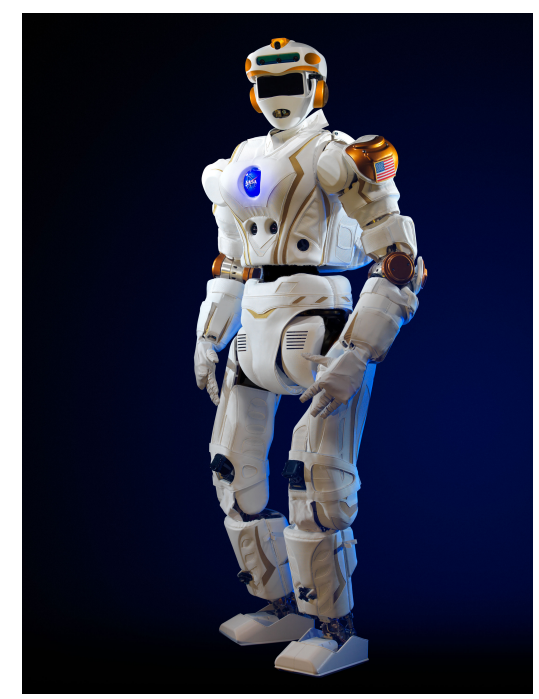

Fig. 1. NASA's Valkyrie humanoid robot 
control, extending this approach to explicitly achieve the aforementioned benefits of an impedance control framework. While [15], [16], and [18] all explore the benefits of DOB based torque (or force) control, the question of impedance rendering accuracy is not specifically addressed. The results presented here fill this gap, demonstrating a DOB's positive impact on SEA impedance rendering and the merit of adopting such an approach for the impedance control of high-performance SEAs in Valkyrie and, by extension, other robots required to accurately render desired dynamic behaviors.

\section{SYSTEM ARCHITECTURE}

\section{A. Plant Model}

A single Valkyrie series elastic actuator (Fig. 2) can be represented by the SEA model illustrated in Fig. 3. Here, motor inertia, $J_{\mathrm{m}}$, and load inertia, $J_{\mathrm{L}}$, are separated by a series elastic spring rate $k$, and are subject to viscous damping values $b_{\mathrm{m}}$ and $b_{\mathrm{L}}$ respectively. The SEA is treated as a dual input system with motor current, $i_{\mathrm{m}}$, as the command input, and external torque, $\tau_{\text {ext }}$, representing exogenous inputs from the environment (e.g. human interaction forces). With $\theta_{\mathrm{m}}$ and $\theta_{\mathrm{L}}$ representing motor and output position respectively, the system dynamics are expressed as:

$$
\begin{gathered}
J_{\mathrm{m}} \ddot{\theta}_{\mathrm{m}}+b_{\mathrm{m}} \dot{\theta}_{\mathrm{m}}+\frac{1}{N} k\left(\frac{1}{N} \theta_{\mathrm{m}}-\theta_{\mathrm{L}}\right)=k_{\mathrm{t}} i_{\mathrm{m}} \\
J_{\mathrm{L}} \ddot{\theta}_{\mathrm{L}}+b_{\mathrm{L}} \dot{\theta}_{\mathrm{L}}-k\left(\frac{1}{N} \theta_{\mathrm{m}}-\theta_{\mathrm{L}}\right)=\tau_{\mathrm{ext}}
\end{gathered}
$$

where $N$ represents the actuator gear ratio and $k_{\mathrm{t}}$ is the motor's torque constant. From (1) and (2) the open loop transfer functions from $i_{\mathrm{m}}$ and $\tau_{\mathrm{ext}}$ to actuator output position $\theta_{\mathrm{L}}$ and measured spring torque,

$$
\tau_{k}=k\left(\frac{1}{N} \theta_{\mathrm{m}}-\theta_{\mathrm{L}}\right)
$$

are derived as:

$$
\begin{gathered}
U(s)=\frac{\theta_{\mathrm{L}}(s)}{i_{\mathrm{m}}(s)}=\frac{N k_{\mathrm{t}} k}{D(s)} \\
V(s)=\frac{\theta_{\mathrm{L}}(s)}{\tau_{\mathrm{ext}}(s)}=\frac{N^{2} J_{\mathrm{m}} s^{2}+N^{2} b_{\mathrm{m}} s+k}{D(s)} \\
W(s)=\frac{\tau_{k}(s)}{i_{\mathrm{m}}(s)}=\frac{N J_{\mathrm{L}} k_{\mathrm{t}} k s^{2}+N b_{\mathrm{L}} k_{\mathrm{t}} k s}{D(s)} \\
X(s)=\frac{\tau_{k}(s)}{\tau_{\mathrm{ext}}(s)}=\frac{-N^{2} J_{\mathrm{m}} k s^{2}-N^{2} b_{\mathrm{m}} k s}{D(s)}
\end{gathered}
$$

where

$$
\begin{aligned}
D(s)=N^{2} & J_{\mathrm{m}} J_{\mathrm{L}} s^{4}+\left(N^{2} J_{\mathrm{m}} b_{\mathrm{L}}+N^{2} b_{\mathrm{m}} J_{\mathrm{L}}\right) s^{3} \\
& +\left(k J_{\mathrm{L}}+N^{2} b_{\mathrm{m}} b_{\mathrm{L}}+N^{2} J_{\mathrm{m}} k\right) s^{2}+\left(k b_{\mathrm{L}}+N^{2} b_{\mathrm{m}} k\right) s .
\end{aligned}
$$

Measurements of $\theta_{\mathrm{L}}$ and $\tau_{k}$ are used as feedback in the Valkyrie impedance control architecture. Thus, the transfer functions (4) - (7) are used in Section II-C to incorporate the plant into the mathematical model of the closed loop actuator used for subsequent analysis and simulation.

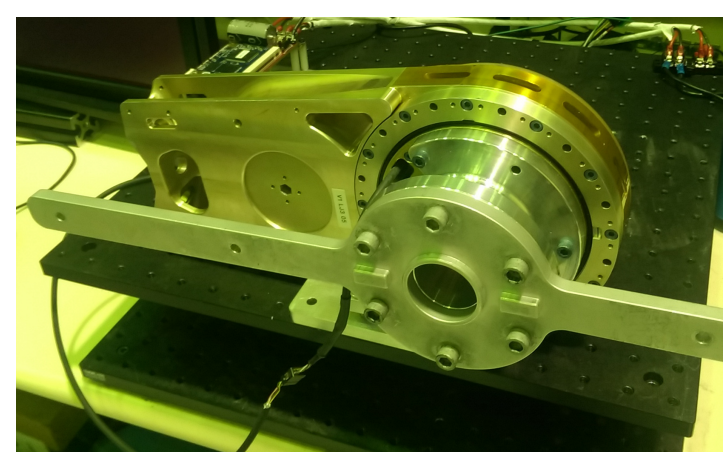

Fig. 2. The Valkyrie hip joint series elastic actuator used for experimentation. It is mounted as a single axis testbed with a lever arm attached at the actuator output for the experiments of Section III.

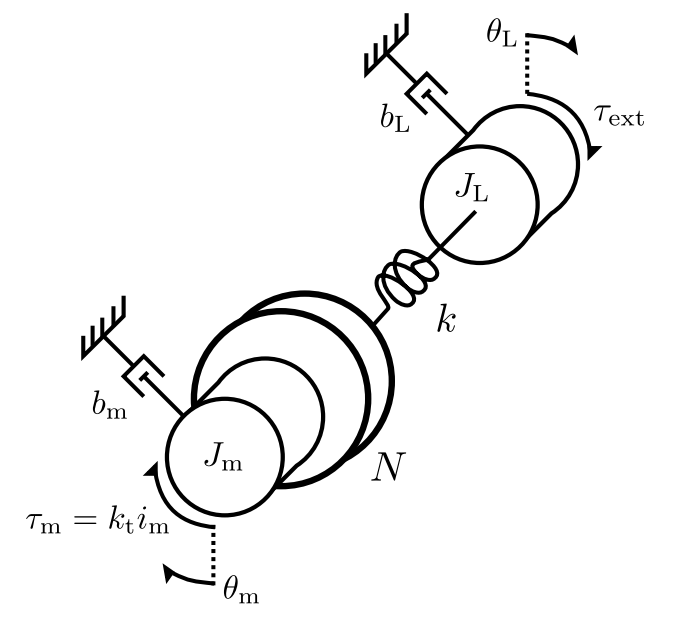

Fig. 3. The series elastic actuator model. $J_{\mathrm{m}}$ and $J_{\mathrm{L}}$ are motor and load inertia respectively. $b_{\mathrm{m}}$ and $b_{\mathrm{L}}$ are motor and output viscous damping. $\theta_{\mathrm{m}}$ and $\theta_{\mathrm{L}}$ are motor and output positions. $k$ is the series elastic spring rate, $N$ is the gear ratio, $\tau_{\mathrm{ext}}$ is the external torque from the environment, and $\tau_{\mathrm{m}}$ is the applied motor torque, a product of motor torque constant $k_{\mathrm{t}}$ and applied motor current $i_{\mathrm{m}}$.

\section{B. Disturbance Observer Based Torque Control}

Valkyrie's disturbance observer based torque control is adapted from an approach outlined in [16] and is described at greater length than space permits here in [18]. It consists of an inner PD compensator acting on torque feedback,

$$
T(s)=K_{\mathrm{dt}} s+K_{\mathrm{pt}},
$$

and a feedforward term, $F F=\left(N k_{\mathrm{t}}\right)^{-1}$. These two components are then combined with the DOB itself as illustrated in the Torque Control section of Fig. 4.

The disturbance observer is constructed from a nominal closed loop plant assuming a fixed output SEA (i.e. an open loop transfer function, $P(s)=\lim _{J_{\mathrm{L}} \rightarrow \infty} W(s)$ ). Combining this open loop plant with $T(s)$ and $F F$ yields an ideal closed loop transfer function from reference torque to actuator spring torque, $P_{\mathrm{n}}(s)$, that is inverted for use in the DOB:

$$
P_{\mathrm{n}}^{-1}(s)=\frac{N^{2} J_{\mathrm{m}} s^{2}+\left(N^{2} b_{\mathrm{m}}+N k_{\mathrm{t}} k K_{\mathrm{dt}}\right) s+\left(k+N k_{\mathrm{t}} k K_{\mathrm{pt}}\right)}{\left(N k_{\mathrm{t}} k K_{\mathrm{dt}}\right) s+\left(k+N k_{\mathrm{t}} k K_{\mathrm{pt}}\right)}
$$




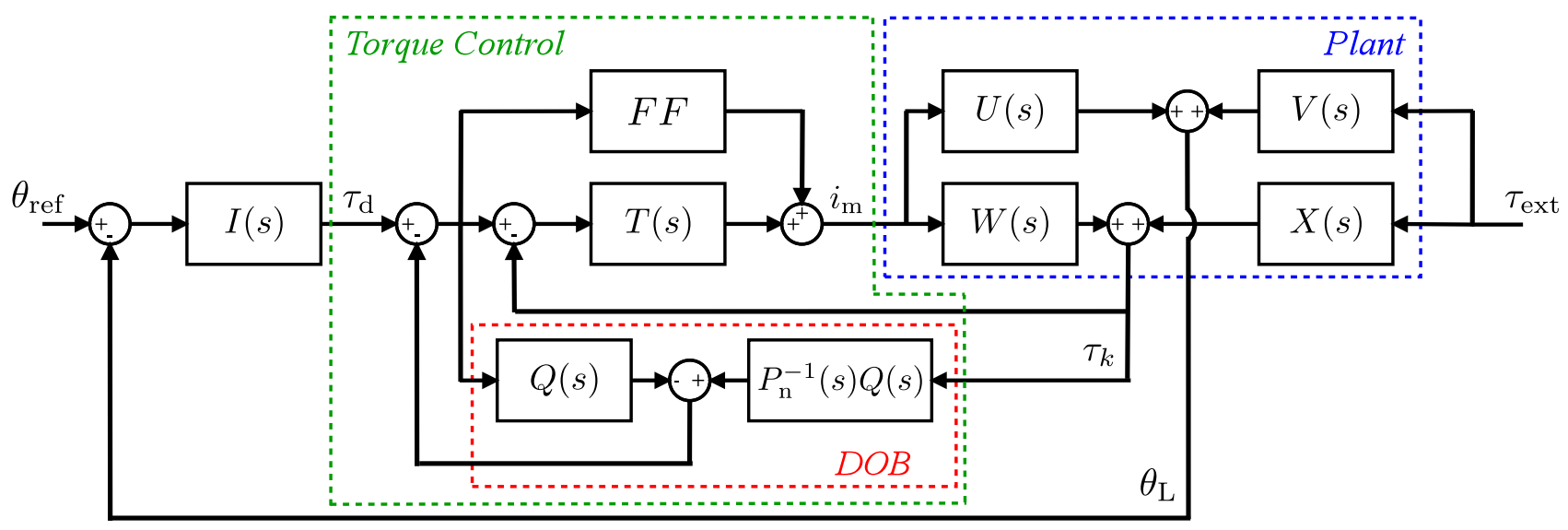

Fig. 4. Impedance control architecture used in the Valkyrie series elastic actuator.

All terms in (9) are either known control gains or determined via experimental system identification of the fixed output actuator.

Also included as part of the DOB is a second order, lowpass Butterworth filter,

$$
Q(s)=\frac{1}{\left(1 / \omega_{\mathrm{q}}^{2}\right) s^{2}+\left(1.4142 / \omega_{\mathrm{q}}\right) s+1},
$$

with cutoff frequency, $\omega_{\mathrm{q}}$. This ensures proper causality of the inverse plant model while also defining the bandwidth over which the DOB is effective. In practice, the torque PD compensator (and the desired impedance PD controller to be discussed later) must also incorporate filtering for their derivative terms. However, these filters have significantly higher cutoff frequencies (in this case $250 \mathrm{~Hz}$ ) than the dynamics of interest. Thus, they are omitted here for the sake of clarity without affecting the presented results.

\section{Closed Loop Model}

The performance experiments in Section III demonstrate that leveraging a DOB in an SEA's inner torque loop has a significant impact on impedance control performance and the fidelity with which desired dynamic behaviors are rendered. The impedance control architecture used for these tests is outlined in full by Fig. 4. Here, DOB based torque control is cascaded with a PD impedance compensator,

$$
I(s)=K_{\mathrm{di}} s+K_{\mathrm{pi}},
$$

and then combined with the plant model of Section II-A.

The gains $K_{\mathrm{pi}}$ and $K_{\mathrm{di}}$ represent the desired spring rate and damping coefficient of the actuator's rendered dynamics, while apparent load inertia is left unmodified by this control approach. As such, the desired output position response to external torque is defined by the $2^{\text {nd }}$ order system:

$$
{\frac{\theta_{\mathrm{L}}(s)}{\tau_{\mathrm{ext}}(s)}}_{\mathrm{des}}=\frac{1}{J_{\mathrm{L}} s^{2}+K_{\mathrm{di}} s+K_{\mathrm{pi}}},
$$

and the desired output position response to reference position commands is similarly defined by:

$$
{\frac{\theta_{\mathrm{L}}(s)}{\theta_{\mathrm{ref}}(s)}}_{\mathrm{des}}=\frac{K_{\mathrm{di}} s+K_{\mathrm{pi}}}{J_{\mathrm{L}} s^{2}+K_{\mathrm{di}} s+K_{\mathrm{pi}}} .
$$

The actual closed loop system responses to external torques and reference commands can be derived directly from the architecture in Fig. 4. Omitting for clarity the explicit dependence on $s$ of each component yields:

$$
\begin{gathered}
\frac{\theta_{\mathrm{L}}(s)}{\tau_{\mathrm{ext}}(s)_{\mathrm{CL}}}=\frac{(V W-U X)\left[P_{\mathrm{n}}^{-1} Q \beta+T \alpha\right]+V \alpha}{\left(P_{\mathrm{n}}^{-1} Q W+U I\right) \beta+(T W+1) \alpha} \\
{\frac{\theta_{\mathrm{L}}(s)}{\theta_{\mathrm{ref}}(s)}}_{\mathrm{CL}}=\frac{U I \beta}{\left(P_{\mathrm{n}}^{-1} Q W+U I\right) \beta+(T W+1) \alpha}
\end{gathered}
$$

where

$$
\begin{gathered}
\alpha=(1-Q) \\
\beta=(F F+T) .
\end{gathered}
$$

Thus defined, (14) and (15) provide a realistic mathematical model of DOB based SEA impedance control enabling both control design and analysis in simulation.

Evidenced by the equivalent denominators of these closed loop transfer functions, both the driving point response to external torque and the forward response to reference position commands exhibit the same stability range. Furthermore, the closed loop poles of each response are affected by plant dynamics and the selection of both torque control and impedance control parameters.

\section{ImPedance Control Performance}

The balance of this paper details experiments performed using a Valkyrie hip joint SEA, demonstrating that inclusion of a DOB in the presented impedance control architecture contributes to better closed loop approximations of desired dynamic behaviors. Both the forward response to a reference position input and the rendered driving point impedance at the actuator's output are improved. In the latter case, improved rendering of desired impedance corresponds to a 
TABLE I

SYSTEM PARAMETERS OF THE VALKYRIE HIP SEA

\begin{tabular}{|c|l|c||c|l|c|}
\hline \multicolumn{4}{|c|}{ Model } & \multicolumn{3}{c|}{ Torque Control } \\
\hline \hline$J_{\mathrm{m}}$ & 0.00013 & $\mathrm{kgm}^{2}$ \\
\hline$b_{\mathrm{m}}$ & 0.0076 & $\mathrm{Nms} / \mathrm{rad}$ & $K_{\mathrm{pt}}$ & 1.0 & $\mathrm{~A} / \mathrm{Nm}$ \\
\hline$J_{\mathrm{L}}$ & 0.15 & $\mathrm{kgm}^{2}$ & $K_{\mathrm{dt}}$ & 0.0143 & $\mathrm{As} / \mathrm{Nm}$ \\
\hline$b_{\mathrm{L}}$ & 0.0 & $\mathrm{Nms} / \mathrm{rad}$ \\
\hline$k$ & 3700 & $\mathrm{Nm} / \mathrm{rad}$ \\
\hline$k_{\mathrm{t}}$ & 0.05 & $\mathrm{Nm} / \mathrm{A}$ \\
\hline$N$ & 120 & - & & \\
& \multicolumn{2}{|c}{} \\
\hline
\end{tabular}

better "feel" when interacting with the SEA, an important consideration for human-centric robots.

The following tests are conducted both with and without the disturbance observer active in the control system. The behavior in each case is compared, and the benefits of the DOB are discussed. The control architecture of Fig. 4 is implemented on a custom, embedded motor controller and runs at $5 \mathrm{kHz}$. For reference, system parameters of the Valkyrie SEA used in testing are summarized in Table I.

\section{A. An Alternative to Higher Gains}

In many control architectures, increasing compensator gains is the easiest way to improve the rendering of desired ideal behaviors. In practice, however, this is not always possible because gain magnitude (particularly that of derivative gains) is fundamentally limited by system nonidealities (e.g. sensor noise, quantization, etc.). If it is assumed that torque gains in the controller $T(s)$ are tuned to acceptable (or perhaps maximum) practical values a priori, the introduction of a DOB serves to improve system performance as if these control gains were increased further.

Fig. 5 illustrates this point with a simulated SEA response to a reference position step command. Without a DOB, the critically damped torque gains $\left(K_{\mathrm{pt}}=1.0 \mathrm{~A} / \mathrm{Nm}\right.$ and $K_{\mathrm{dt}}=$ $0.014 \mathrm{As} / \mathrm{Nm}$ ) are seen to have a significantly slower rise time than the desired dynamic behavior $(0.42 \mathrm{~s}$ vs. $0.029 \mathrm{~s})$, causing the actuator to appear sluggish in its response. If possible, an increase of $K_{\mathrm{pt}}$ to $5.0 \mathrm{~A} / \mathrm{Nm}$ (and a corresponding increase of $K_{\mathrm{dt}}$ to maintain the critically damped nature of the controller) would provide a much better match to the desired behavior. This, as mentioned however, might not be an option in practice. By including a DOB in the controller with the original torque gains, improved performance is achieved without requiring a gain increase. The DOB, in fact, provides a comparable settling time, a comparable peak tracking error of the desired dynamic response, and a faster rise time $(0.042 \mathrm{~s}$ vs. $0.080 \mathrm{~s})$, with respect to the previous factor of five increase in torque compensator gain.

\section{B. Step Responses}

Experimental tests of the Valkyrie SEA's response to a $0.05 \mathrm{rad}$ reference position step yields Fig. 6. Here, the desired virtual stiffness and damping are $K_{\mathrm{pi}}=100 \mathrm{Nm} / \mathrm{rad}$

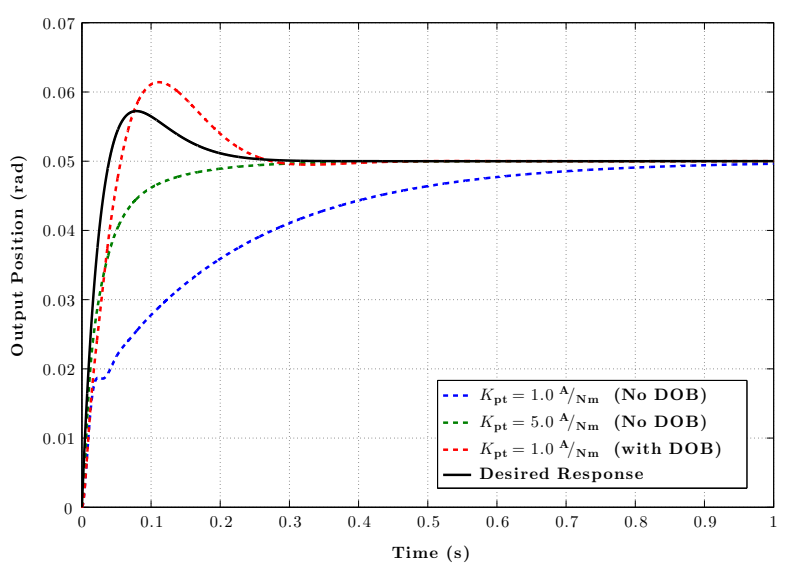

Fig. 5. Simulated response to a $0.05 \mathrm{rad}$ step in reference position given a desired virtual stiffness and damping of $K_{\mathrm{pi}}=100 \mathrm{Nm} / \mathrm{rad}$ and $K_{\mathrm{di}}=7.36 \mathrm{Nms} / \mathrm{rad}$. The DOB provides a comparable (if not preferred) match to the desired dynamic response, compared to a factor of five increase in torque compensator proportional gain.

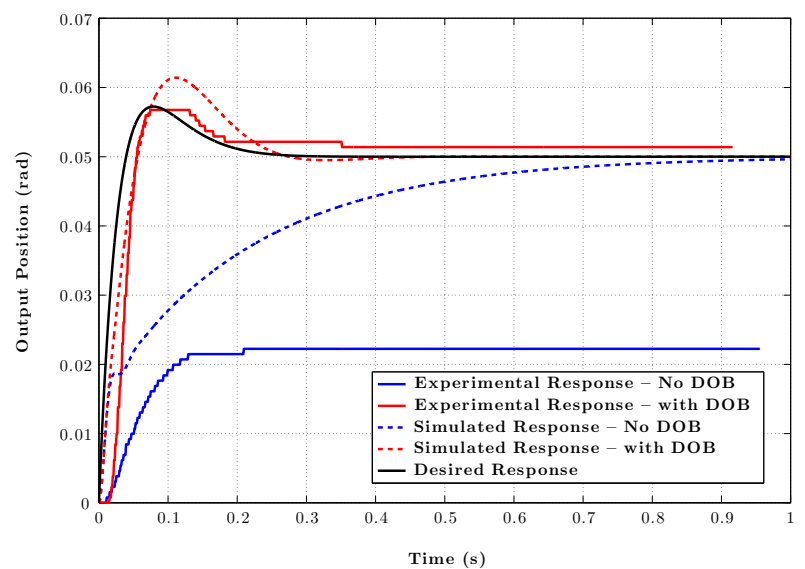

Fig. 6. Experimental response to a $0.05 \mathrm{rad}$ step in reference position given a desired virtual stiffness and damping of $K_{\mathrm{pi}}=100 \mathrm{Nm} / \mathrm{rad}$ and $K_{\mathrm{di}}=$ $7.36 \mathrm{Nms} / \mathrm{rad}$. Note the significant improvements in rise time and steady state error achieved with a DOB.

and $K_{\mathrm{di}}=7.36 \mathrm{Nms} / \mathrm{rad}$, while the torque control gains are held constant according to Table I.

Compared to the controller with no DOB (solid blue line), DOB based control (solid red line) provides a much closer approximation of the ideal desired response. This is true for all meaningful transient response measures, as is readily apparent in Fig. 6. Of particular interest in the experimental data, a large steady state error is observed in the test with no disturbance observer. Neither the model developed for simulation in Section II, nor the controller, have explicit knowledge of friction in the actuator (the likely cause of this steady state error). Because the DOB acts to enforce the nominal closed loop plant, it serves to mitigate the effects of unmodeled friction, highlighting an important strength of DOB based control. In spite of this difference, the experimental data exhibit the same qualitative behavior as their corresponding simulated responses (this is particularly obvious in the transient behaviors prior to time $0.2 \mathrm{~s}$ ). Thus, greater confidence can be placed in conclusions drawn from 


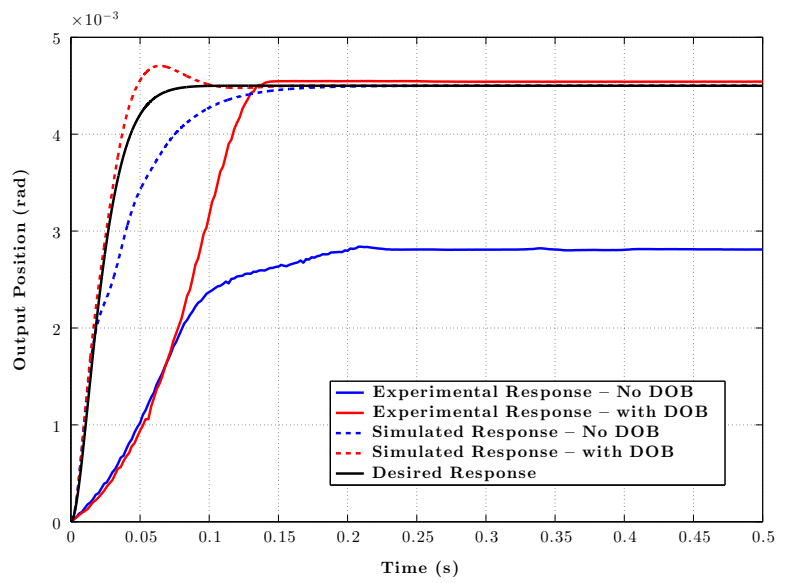

Fig. 7. Experimental response to a $4.5 \mathrm{Nm}$ step in external torque given a desired virtual stiffness and damping of $K_{\mathrm{pi}}=1000 \mathrm{Nm} / \mathrm{rad}$ and $K_{\mathrm{di}}=$ $23.27 \mathrm{Nms} / \mathrm{rad}$. Note the DOB's ability to eliminate steady state error on the physical actuator.

model based analysis.

Performance improvements due to the DOB are observed in the SEA's response to external torques as well. In Fig. 7, a critically damped, desired virtual stiffness and damping of $K_{\mathrm{pi}}=1000 \mathrm{Nm} / \mathrm{rad}$ and $K_{\mathrm{di}}=23.27 \mathrm{Nms} / \mathrm{rad}$ is set. For this experiment, however, the desired reference position is held at zero and a $1.8 \mathrm{~kg}$ mass is dropped on a $0.25 \mathrm{~m}$ lever arm attached to the actuator output. This produces a $4.5 \mathrm{Nm}$ step in external torque. Again, a large reduction in steady state error due to the DOB is apparent in Fig. 7. As both transient response and steady state deflection are important for accurate virtual dynamics, it is clear leveraging a DOB in control significantly improves impedance rendering.

It should be noted that applying an external torque with a dropped mass results in a less than ideal input step. This leads to the slower transients observed in the experimental data. Otherwise, the results in Fig. 7 again confirm the predicted behavior from the mathematical model and the benefit of using a DOB in impedance control.

\section{Low Impedance Rendering}

Another important benchmark for impedance controlled actuators is the ability to render very low impedances. Device transparency is particularly desirable for systems designed to interact with people (e.g. haptic displays, rehabilitation robots, and exoskeletons) and it serves as a lower bound on the effective impedance range that an actuator can render. The plots of Fig. 8 show the results of an experiment in which the Valkyrie SEA was commanded to render a zero impedance $\left(K_{\mathrm{pi}}=K_{\mathrm{di}}=0\right)$. A person then disturbed the actuator output by moving an attached lever arm while both the actuator output position (Fig. 8(a)) and torque (Fig. 8(b)) were recorded. The magnitude of torque felt at the output (zero being ideal) serves as a measure of device transparency.

From Fig. 8(b) it is clear that including a DOB in the control system greatly enhances the actuator's ability to faithfully render near zero impedances. With the DOB active, the peak torque magnitude felt by the person is reduced from

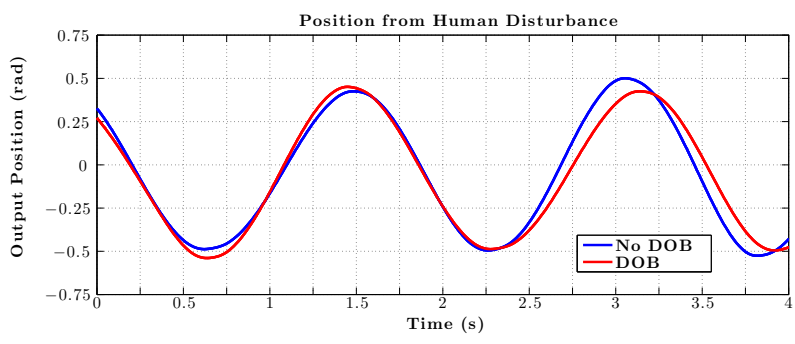

(a)

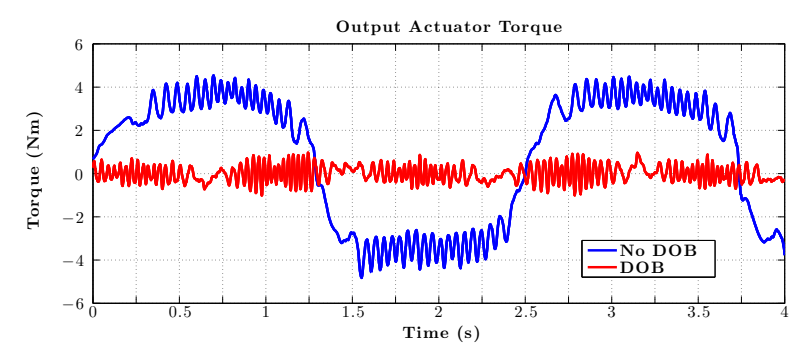

(b)

Fig. 8. Device transparency test. (a) The disturbance position introduced by a person interacting with the SEA output and (b) the resulting actuator torque felt by the user when the SEA is commanded to render a zero impedance. The DOB provides a significant improvement in actuator transparency, decreasing peak output torque magnitude from $4.8 \mathrm{Nm}$ to $1.0 \mathrm{Nm}$.

$4.8 \mathrm{Nm}$ to $1.0 \mathrm{Nm}$ during the roughly $0.6 \mathrm{~Hz}$ position sinusoid being input.

\section{Reduced Actuator Hysteresis}

Another measure of impedance control performance, less commonly explored than the low impedance tests of the previous section, is actuator hysteresis. Due to its reliance on an intentionally compliant element, a series elastic actuator can be particularly susceptible to hysteresis in both torque measurement and controlled response if careful attention is not paid to the mechanical design and mounting of the passive spring and its deflection sensor. This is especially true for high torque actuators, like those in Valkyrie, that are expected to exhibit small controllable torque resolutions (minimum torque resolutions as low as $0.002 \mathrm{Nm}$ are demonstrated for some Valkyrie joints in [18]).

From an impedance rendering perspective, hysteresis hurts performance by preventing a consistent, repeatable, one-toone mapping of applied external torque to actuator output position. Qualitatively, it also degrades the haptic sensation experienced when interacting with the actuator. Potential sources of hysteresis in Valkyrie SEAs include friction, deflection in structural elements, and the harmonic drive gear train, all of which are difficult to quantify and model. Thus, a DOB's ability to enforce nominal plant dynamics is beneficial.

DOBs have been shown to reduce hysteresis in piezoelectric actuators [19], and indeed, as Fig. 9 demonstrates, they also serve this purpose in impedance controlled SEAs. Once again relying on human input, the actuator output is flexed repeatedly between $30 \mathrm{Nm}$ and $-30 \mathrm{Nm}$ while a virtual 


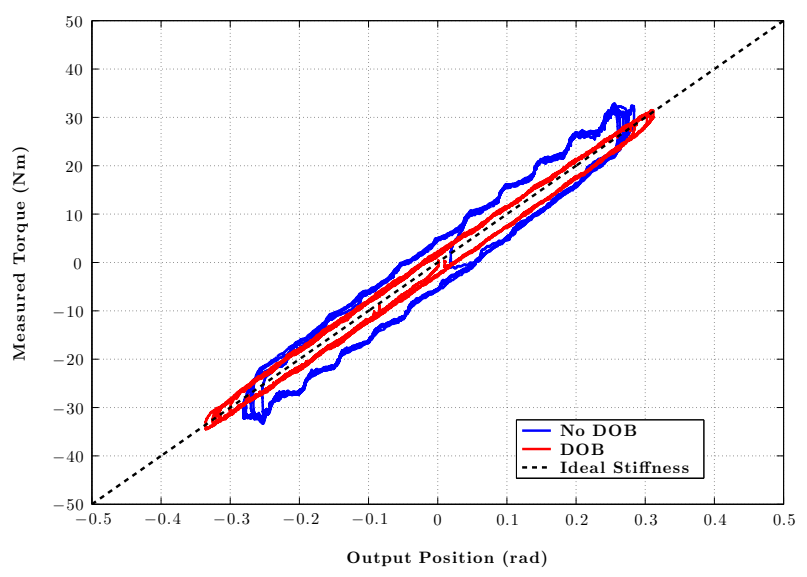

Fig. 9. Hysteresis exhibited by the Valkyrie SEA while rendering a virtual stiffness of $K_{\mathrm{pi}}=100 \mathrm{Nm} / \mathrm{rad}$. Torque data is taken from an external load cell while the actuator output is deflected by a human user. The DOB provides a more accurate match to the ideal stiffness and decreases the width of the hysteresis curve by over $50 \%$.

stiffness of $K_{\mathrm{pi}}=100 \mathrm{Nm} / \mathrm{rad}$ is commanded. The dashed black line in the figure represents the ideal rendered stiffness, while the other curves represent the output torque during motion, as measured by an external load cell. The blue line is without a DOB while the red line has the DOB active.

Two things are readily apparent from Fig. 9. First, when the DOB is active it serves to realign the actuator's rendered stiffness with the desired ideal value. In light of a DOB's ability to enforce nominal plant dynamics, this makes sense. Second, the disturbance observer significantly reduces the width of the hysteresis curve. Around zero position the controller without a DOB exhibits an $11 \mathrm{Nm}$ torque range, while the DOB decreases this hysteresis error by more than half, to only $5 \mathrm{Nm}$. Thus, leveraging DOB based torque control yields an SEA capable of rendering desired impedances more accurately.

\section{CONCLUSION}

The fidelity with which SEAs render desired impedances is important. Actuators used in haptic displays must "feel" right to the user, wearable robots must provide transparency when commanded to do so, and humanoid robots such as NASA's Valkyrie must reliably modulate impedance to perform human-like tasks and interactions. The system model constructed in Section II provides a mathematical basis for exploring SEA impedance control performance. Specifically, future work will utilize this model to examine the full range over which impedance controlled SEAs are stable and passive when DOB based torque control is used, as in Valkyrie.

Leveraging DOB based torque control for impedance rendering has a number of demonstrable benefits. Transparency is improved, actuator hysteresis is reduced, and the dynamic response to both reference commands and external torques more faithfully matches that of a desired virtual impedance. This is all achieved by incorporating a DOB in the control architecture, rather than relying on increased compensator gains, a significant benefit in practical applications. Experimental results speak to the merit of using DOB based torque control to realize improved impedance rendering with SEAs, and continued research in this arena will likely lead to additional improvements in SEA performance during interaction tasks.

\section{REFERENCES}

[1] N. Hogan, "Impedance control: An approach to manipulation," Journal of Dynamic Systems, Measurement, and Control, vol. 107, no. 1, pp. 1-24, Mar. 1985.

[2] D. W. Franklin, R. Osu, E. Burdet, M. Kawato, et al., "Adaptation to stable and unstable dynamics achieved by combined impedance control and inverse dynamics model," Journal of Neurophysiology, vol. 90, no. 5, pp. 3270-3282, 2003.

[3] N. Hogan, "Adaptive control of mechanical impedance by coactivation of antagonist muscles," IEEE Trans. Automat. Contr., vol. AC-29, no. 8, pp. 681-690, Aug. 1984.

[4] G. A. Pratt and M. M. Williamson, "Series elastic actuators," in Proc. IEEE/RSJ Int. Conf. Intelligent Robots and Systems, Aug. 1995, pp. 399-406.

[5] J. Pratt, B. Krupp, and C. Morse, "Series elastic actuators for high fidelity force control," Industrial Robot: An International Journal, vol. 29, no. 3, pp. 234-241, 2002.

[6] D. Paluska and H. Herr, "The effect of series elasticity on actuator power and work output: Implications for robotic and prosthetic joint design," Robotics and Autonomous Systems, vol. 54, pp. 667-673, 2006.

[7] M. Zinn, O. Khatib, B. Roth, and J. K. Salisbury, "Large workspace haptic devices - a new actuation approach," in Proc. IEEE Haptics Symposium, March 2008, pp. 185-192.

[8] F. Sergi, M. M. Lee, and M. K. O'Malley, "Design of a series elastic actuator for a compliant parallel wrist rehabilitation robot," in Proc. IEEE Int. Conf. Rehabilitation Robotics, June 2013, pp. 1-6.

[9] H. Vallery, J. Veneman, E. van Asseldonk, R. Ekkelenkamp, et al., "Compliant actuation of rehabilitation robots," IEEE Robot. Automat. Mag., vol. 15, no. 3, pp. 60-69, Sept. 2008.

[10] D. W. Robinson, "Design and analysis of series elasticity in closedloop actuator force control," Ph.D. dissertation, Massachusetts Institute of Technology, 2000.

[11] G. Pratt, P. Willisson, C. Bolton, and A. Hofman, "Late motor processing in low-impedance robots: impedance control of serieselastic actuators," in Proc. American Control Conference, vol. 4, June 2004, pp. 3245-3251.

[12] J. Sensinger and R. Weir, "Improvements to series elastic actuators," in Proc. IEEE/ASME Int. Conf. Mechatronic and Embedded Systems and Applications, Aug 2006, pp. 1-7.

[13] H. Vallery, R. Ekkelenkamp, H. van der Kooij, and M. Buss, "Passive and accurate torque control of series elastic actuators," in Proc. IEEE/RSJ Int. Conf. Intelligent Robots and Systems, Oct 2007, pp. 3534-3538.

[14] N. L. Tagliamonte and D. Accoto, "Passivity constraints for the impedance control of series elastic actuators," Proc. of the Institution of Mechanical Engineers, Part I: Journal of Systems and Control Engineering, vol. 228, no. 3, pp. 138-153, Mar. 2014.

[15] K. Kong, J. Bae, and M. Tomizuka, "Control of rotary series elastic actuator for ideal force-mode actuation in human-robot interaction applications," IEEE/ASME Trans. Mechatron., vol. 14, no. 1, pp. 105118, Feb. 2009.

[16] N. Paine, S. Oh, and L. Sentis, "Design and control considerations for high-performance series elastic actuators," IEEE/ASME Trans. Mechatron., vol. 19, no. 3, pp. 1080-1091, June 2014.

[17] M. A. Diftler, J. S. Mehling, M. E. Abdallah, N. A. Radford, et al., "Robonaut 2 - the first humanoid robot in space," in Proc. IEEE Int. Conf. Robotics and Automation, May 2011, pp. 2178-2183.

[18] N. Paine, J. S. Mehling, J. Holley, N. Radford, et al., "Actuator control for the NASA-JSC Valkyrie humanoid robot: A decoupled dynamics approach for torque control of series elastic robots," Journal of Field Robotics, vol. 32, no. 3, pp. 378-396, May 2015.

[19] J. Yi, S. Chang, and Y. Shen, "Disturbance-observer-based hysteresis compensation for piezoelectric actuators," IEEE/ASME Trans. Mechatron., vol. 14, no. 4, pp. 456-464, Aug 2009. 